\title{
Desensitization to dyspnea in COPD with specificity for exercise training mode
}

\section{Christopher B Cooper}

David Geffen School of Medicine, University of California, Los Angeles, Los Angeles, CA, USA
Correspondence: Christopher B Cooper David Geffen School of Medicine, University of California, Los Angeles, 10833 Le Conte Avenue, 37-13 I CHS, Los Angeles, CA 90095-1690, USA

Tel + I 3108254440

Fax + I 3102068211

Email ccooper@mednet.ucla.edu

\begin{abstract}
Patients with chronic obstructive pulmonary disease (COPD) exhibit improved exercise capacity after physical training due to reconditioning and improved ventilatory efficiency. Other possible effects are improved ventilatory muscle function and desensitization to dyspnea. We compared general physical training (GPT), consisting of walking and stair climbing exercises, with inspiratory muscle training (IMT), consisting of targeted breathing through inspiratory resistances, in two groups with severe COPD. Seven subjects; age 60 (8) years, forced expiratory volume in one second $\left(\mathrm{FEV}_{1}\right) 0.84(0.35) \mathrm{L}$, arterial oxygen tension $\left(\mathrm{PaO}_{2}\right) 11.1(0.8) \mathrm{kPa}$, arterial carbon dioxide tension $\left(\mathrm{PaCO}_{2}\right) 4.9(0.3) \mathrm{kPa}$, had GPT and nine subjects; age 60 (9) years, $\mathrm{FEV}_{1} 0.83$ (0.31) L, $\mathrm{PaO}_{2} 10.4(0.8) \mathrm{kPa}, \mathrm{PaCO}_{2} 4.4(0.5) \mathrm{kPa}$ had IMT. Each group trained daily for 30 minutes for eight weeks and $70 \%$ of the sessions were supervised. Six minute walking distance increased in both groups: $32 \mathrm{~m}(6.9 \% \mathrm{P}<0.05)$ with GPT and $23 \mathrm{~m}(4.8 \%$; P $<0.05)$ with IMT but significant improvements in symptom-limited incremental cycle exercise performance were not detected. Breathlessness by visual analog scale was reduced following 6-minute walks after GPT $(\mathrm{P}<0.05)$ but not after IMT. Following maximal incremental tests, breathlessness scores were unchanged for both groups. COPD patients performing regular physical exercise report reductions in breathlessness which are specific to the exercise training mode.
\end{abstract}

Keywords: COPD, exercise, ventilatory muscles, dyspnea

\section{Introduction}

Patients with chronic obstructive pulmonary disease (COPD) typically complain of dyspnea and reduced exercise capacity. Randomized controlled trials have shown an improvement in exercise capacity by a structured schedule of physical exercise. ${ }^{1,2}$ Two mechanisms have been shown to contribute to this improvement, including improved physical reconditioning (a true physiological training effect) ${ }^{3}$ and improved ventilatory efficiency. ${ }^{4}$ Two other mechanisms that might be implicated are improved ventilatory muscle function and desensitization to dyspnea.

A recent study ${ }^{5}$ has shown that COPD patients have reduced daily physical activity and this is thought to contribute to the development of deconditioning (sustained increases in blood lactate at relatively low levels of exercise compared with normal subjects). ${ }^{6}$ One training study in COPD patients demonstrated reductions in exercise lactic acidosis and ventilation as a result of exercise training. ${ }^{3}$ These investigators attributed the related improvements in endurance for cycle ergometer exercise to a reduced ventilatory requirement. Other investigators have studied the potential role of respiratory muscle training in improving ventilatory capacity. Several studies have reported improvements in respiratory muscle endurance but convincing evidence of improved exercise capacity has not been demonstrated. ${ }^{7}$ In a controlled study of COPD patients, Dekhuijzen and colleauges ${ }^{8}$ showed that the addition of target-flow inspiratory muscle training to a pulmonary rehabilitation program resulted in better 
inspiratory muscle strength and resistance to fatigue but again the benefits in terms of exercise capacity were marginal.

Desensitization to dyspnea is often discussed as a mechanism to explain benefit in the rehabilitation of COPD patients but few studies have systematically examined this phenomenon. Altered perception of breathlessness may occur as a separate entity from other physiological changes. A common hypothesis used to explain the mechanism is alleviation of fear by repetitive performance of exercise tasks in a safe and supervised environment. ${ }^{9}$ This mechanism might be expected to show some specificity for the type of exercise involved. Another suggestion is that techniques of respiratory muscle training might alter perception of dyspnea rather than ventilatory capacity. ${ }^{10}$ Despite many studies of physical training in COPD in recent years, the symptomatic aspects of COPD and rehabilitation lend themselves to further investigation.

We set out to compare two training techniques commonly used in pulmonary rehabilitation, examining both physiological and symptomatic differences between the two groups to distinguish between possible mechanisms of improvement. Subjects with clinically stable COPD were randomly allocated to receive either general physical training (GPT) supervised by a registered physical therapist or targeted inspiratory muscle training (IMT) supervised by a physician. The outcome after four and eight weeks was assessed in terms of pulmonary function tests, exercise capacity, respiratory muscle strength, and symptom scores.

\section{Methods}

\section{Subjects}

The subjects selected for this study fulfilled the following criteria: (a) symptoms consistent with the definition of chronic bronchitis by the Medical Research Council Committee on the Aetiology of Chronic Bronchitis, ${ }^{11}$ (b) exercise capacity limited by dyspnea, and (c) absence of other disabling disease including symptomatic ischemic heart disease, peripheral vascular disease or musculoskeletal disorders. The experimental protocol was approved by the Research and Ethical Committee of St. Thomas' Hospital and each subject gave informed written consent. The subjects were recruited whilst in a stable clinical condition and none were studied within three months of hospital admission for an exacerbation of COPD. All subjects had moderately severe, poorly reversible airflow obstruction with a forced expiratory volume in one second $\left(\mathrm{FEV}_{1}\right)$ less than $1.5 \mathrm{~L}$ with less than $15 \%$ improvement after inhalation of albuterol $400 \mu \mathrm{g}$. Nonsmoking subjects were preferred but moderate smokers were included providing that their tobacco consumption appeared to remain constant. Carboxyhemoglobin levels were monitored. The total number of subjects assessed was 57. Twenty-six of these were found to be unsuitable since they did not meet the selection criteria defined above.

\section{Randomization}

Subjects fulfilling the selection criteria were randomly allocated into two groups using a random number table. Fifteen subjects (13 men, 2 women) were allocated to receive GPT. Eight of these were subsequently withdrawn during the study; four had exacerbations of COPD, one had a myocardial infarction and three declined to continue. Seven subjects ( 5 men, 2 women) completed the program of GPT. Their mean age was 60 years (SD 8), with a range of 45 to 68 years, and the $\mathrm{FEV}_{1}$ was $0.84(0.35) \mathrm{L}$. Sixteen subjects (14 men, 2 women) were allocated to IMT. Seven were withdrawn; two had exacerbations of COPD, one was found to have a carcinoid tumour of the lung, one developed cardiac dysrhythmias and three declined to continue. Nine subjects (all male) completed the program of IMT. Their age was 60 (9) years, with a range from 46 to 74 years, and the $\mathrm{FEV}_{1}$ was 0.83 (0.31) L. Both groups of subjects showed hyperinflation and impaired gas transfer for carbon monoxide.

\section{Procedures}

\section{Pulmonary function tests}

The FEV 1 and forced vital capacity (FVC) were measured using a calibrated wedge bellows spirometer (Vitalograph Ltd, Buckingham, UK). FEV ${ }_{1}$ was also expressed as a percentage of the European adult predicted value. ${ }^{12}$ Lung volumes were determined using a whole body plethysmograph (26000 A4; PK Morgan Ltd, Chatteris, UK) and carbon monoxide transfer factor was determined by the single breath technique (Respirometer Mk 4; PK Morgan Ltd). At the beginning of each study day, blood was obtained by radial artery puncture with subjects sitting at rest, breathing air. Gas tensions were determined using a blood gas analyzer (Corning 168; Corning Medical and Scientific Ltd, Halstead, UK). Carboxyhemoglobin levels were measured using a cooximeter (Co-oximeter 282; Instrumentation Laboratories Inc, Tucson, USA).

\section{Six-minute walking tests}

Exercise capacity was measured by six-minute walking tests modeled after a standardized protocol. ${ }^{13}$ These tests were carried out on a level, enclosed hospital corridor and were supervised by the same technician walking behind the subjects. The importance of standardization was recognized 
and therefore instructions were given, before the start of each walking test, in the form of a short tape recording designed to eliminate bias which might be introduced by variation in supervisor encouragement. ${ }^{14}$ Walking distance is a reproducible measure of exercise capacity in COPD once the recognized learning effect has been overcome. ${ }^{15}$ In order to determine the magnitude of the learning effect with this protocol, we measured six-minute walking distance $\left(\mathrm{WD}_{6}\right)$ in a different group of subjects with COPD, on three consecutive days. The results are shown in Figure 1. A significant increase in walking distance occurred between tests 1 and 2 $(8 \%, \mathrm{P}<0.001)$. A further increase in walking occurred between tests 2 and $3(3 \%)$ but this did not reach statistical significance. The difference (11\%) between tests 1 and 3 was highly significant $(\mathrm{P}<0.001)$. On the basis of these findings it was decided that each subject should perform two practice walking tests before the initial assessment.

\section{Incremental exercise tests}

Exercise capacity was also evaluated by incremental exercise testing. The subject was seated on an electrically loaded cycle ergometer (Lode NV Instrumentation, Dayton, USA) with seat and pedals carefully adjusted. A standard mouthpiece was used and the nose was comfortably clipped. After a period of acclimatization to the equipment, stable resting measurements were made. The subject was then instructed to commence pedaling and to maintain a target pedaling frequency of $60 \mathrm{~min}^{-1}$. The work-load was incremented at minute intervals until the subject no longer wished to continue. The rate of increase of work-load was selected to suit each individual subject, $\left(10,15\right.$, or 20 watts $\left.\mathrm{min}^{-1}\right)$ aiming for an exercise phase duration between eight and 12 minutes. ${ }^{16}$ On repeated tests the same increment was chosen for any given subject. Minute ventilation $\left(\dot{V}_{E}\right)$ was measured throughout the test using a low resistance dry gas meter (Parkinson-Cowan 2033) which was calibrated each day using a precision-made one-liter syringe (PK Morgan Ltd). Oxygen uptake $\left(\dot{V} \mathrm{O}_{2}\right)$ and carbon dioxide output $\left(\dot{V} \mathrm{CO}_{2}\right)$ were determined using a mixing chamber system and discrete gas analyzers (Servomex 570A and Gould Capnograph IV). The gas analyzers were calibrated at the beginning and end of each day with precision cylinder gases. Heart rate $\left(f_{c}\right)$ was monitored continuously using surface

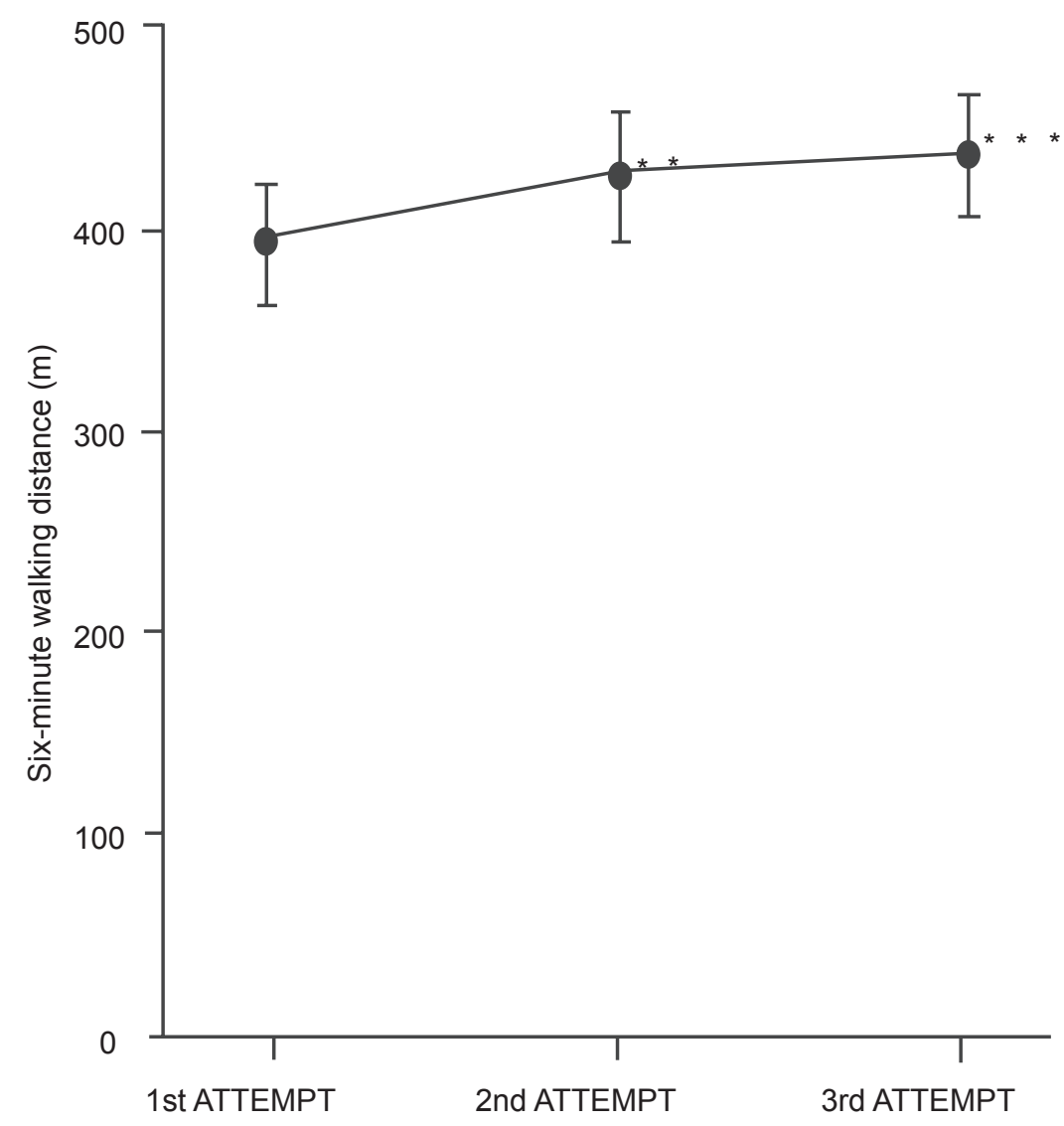

Figure I Reproducibility of six-minute walking tests in 12 subjects with chronic obstructive pulmonary disease over three consecutive days. Values are means with standard error bars. Comparing the Ist and 2 nd attempts, walking distance increased by $8 \%$. Comparing the Ist and 3rd attempts, walking distance increased by $1 \mathrm{I} \%$. Notes: Statistical significance: $* * \mathrm{P}<0.01$, $* * * \mathrm{P}<0.001$. The difference between the 2 nd and $3 \mathrm{rd}$ attempts was not significant. 
electrodes and an electrocardiographic monitor ( $\mathrm{S}$ and $\mathrm{W}$ Diascope DS521). Arterial oxygen saturation $\left(\mathrm{SaO}_{2}\right)$ was monitored using an ear-oximeter (Hewlett-Packard 47201A). Measured values were compared with reference values for normal subjects and the exercise responses were analyzed using a practical approach. ${ }^{17}$ Preliminary studies in a different group of 12 COPD subjects indicated that the incremental exercise tests performed according to these methods were reproducible for individual subjects after familiarization with the equipment. Our findings confirmed a previous report that the learning effect with repeated exercise testing on the cycle ergometer is less than $5 \% .{ }^{18}$ On the basis of these findings it was decided that each subject should perform one practice exercise test on the cycle ergometer prior to entry into the study.

\section{Maximal respiratory pressures}

Respiratory muscle strength was evaluated by recording maximal static expiratory and inspiratory mouth pressures using a pressure transducer (Gaeltec $6 \mathrm{~T}$ ) with a linear range from -200 to $+300 \mathrm{~cm} \mathrm{H}_{2} \mathrm{O}$. The subjects were seated with noses comfortably clipped. They were instructed to seal their lips tightly around a rigid cardboard mouth-piece and apply their hands to their cheeks to prevent perioral leak. They were then instructed to perform a respiratory manoeuvre either to total lung capacity (TLC) for the measurement of maximal expiratory pressure $\left(P_{E} \max \right)$ or to residual volume (RV) for the measurement of maximal inspiratory pressure $\left(P_{I} \max \right)$. The airway was occluded by abrupt manual closure of a wide-bore tap (Cranlea and Co. Ltd, Birmingham, UK) and the subject was instructed to perform a maximal expiratory or inspiratory effort as appropriate. During the measurement of $P_{I}$ max, a $0.6 \mathrm{~mm}$ diameter leak was incorporated in the tubing close to the mouth-piece to ensure that the glottis remained open, thus preventing the generation of spuriously high buccal pressures. Respiratory excursions were monitored using a cylindrical rolling seal spirometer (Ohio 842) so that repeated measurements could be performed at the same lung volume. The signals from the spirometer and pressure transducer were displayed on a twin-channel pen recorder (Devices DC5H). The mean of three technically successful measurements was taken to be $P_{E} \max$ or $P_{I} \max$.

The reproducibility of static mouth pressure measurements by the techniques used in this study was assessed in a different group of 12 practiced subjects with COPD over three consecutive days. The coefficient of variation for $P_{E}$ $\max$ was $6.8 \%$ and for $P_{I} \max 8.2 \%$. These findings are consistent with the results of other investigators. ${ }^{19}$

\section{Symptom assessments}

Immediately after each exercise test, the subjects were given a Borg scale with which to rate perceived exertion. ${ }^{20}$ This category scale with ratio properties is reproducible in patients with COPD and correlates with both minute ventilation and oxygen consumption. ${ }^{21}$ A $100 \mathrm{~mm}$ visual analog scale (VAS) was used to score breathlessness. In the assessment of dyspnea the VAS has been shown to be reproducible and sensitive to changes in subjects with COPD. ${ }^{22}$ The VAS is also simple to use and differences can be treated by parametric statistical tests, within-subject comparisons being more sensitive and accurate than between-subject ones. ${ }^{23}$

\section{Experimental protocol}

After familiarization with the techniques of assessment and adaptation to the equipment, the subjects were evaluated by the methods described and then randomly allocated into two groups. One group performed general physical training whilst the other group trained only the inspiratory muscles. Exercise assessments were performed after four and eight weeks. Full assessments were repeated after eight weeks.

\section{General physical training}

Each subject in the GPT group was instructed to perform graded exercises, walking and climbing stairs for $30 \mathrm{~min}$ utes each day on five days each week. Training sessions were supervised at the hospital or in the subject's home by an experienced physiotherapist. The intensity of exertion was judged by the pulse rate which was monitored during training. Subjects were encouraged to achieve a heart rate of approximately $70 \%$ of their predicted maximum value. The number of stairs climbed or the distance walked was recorded on diary cards.

\section{Inspiratory muscle training}

The subjects in the IMT group trained their inspiratory muscles by breathing through inspiratory resistances and achieving a target mouth pressure. The resistances were mounted on a specially modified, close fitting mask (Hudson Oxygen Mask) which incorporated a one-way valve to permit unimpeded expiration. The inspiratory resistances consisted of interchangeable endotracheal tube connectors (Portex Ltd, Hythe, UK). Their internal diameters and flow resistances were determined as shown in Table 1. The inspiratory resistance was adjusted at the beginning of training and every week thereafter so as to achieve $40 \%$ of $P_{I}$ max with each breath. These adjustments were achieved by having the subjects breathe through the inspiratory resistance whilst mouth pressures were monitored over 15 minutes using the 
Table I Resistance of the adaptors (Portex Ltd) used for inspiratory muscle training

\begin{tabular}{|c|c|}
\hline $\begin{array}{l}\text { Diameter } \\
(\mathrm{mm})\end{array}$ & $\begin{array}{l}\text { Resistance } \\
\left(\mathrm{cm} \mathrm{H} \mathrm{H}_{2} \mathrm{~L}^{-1} \mathrm{~s}\right)\end{array}$ \\
\hline 4.0 & 150 \\
\hline 3.5 & 240 \\
\hline 3.0 & 300 \\
\hline 2.5 & 360 \\
\hline
\end{tabular}

Notes: The pressure flow relationships for the four resistances were curvilinear. Resistance values were calculated within the range of flows $0.75-2.00 \mathrm{~L}^{\mathrm{s}-1}$.

pressure transducer. The mask was worn for 30 minutes each day on five days each week. Subjects were usually seated comfortably, but the apparatus could also be tolerated whilst moving short distances and carrying out simple domestic tasks. Sessions were supervised by one of the investigators either at the hospital or in the subject's home.

\section{Statistical analysis}

Descriptive statistics were expressed as means and standard deviations. Group comparisons were expressed as means and standard errors. The significance of the differences between group means was assessed using repeated measures analysis of variance and student's t-test as appropriate for paired or unpaired data. ${ }^{24}$ Whenever more than one t-test was applied to the same data set, a Bonferroni correction was included to adjust for multiple comparisons. A probability of $5 \%$ or less was considered significant. Coefficients of variation were found to be $<10 \%$ for the primary exercise test parameters and maximal respiratory pressures in a comparable group of subjects. A priori, we considered that a $15 \%$ change in any of these parameters, occurring as a result of the training intervention, would represent a clinically meaningful difference. Power analysis based on these considerations would give a $90 \%$ chance of detecting a clinically meaningful change with groups of ten subjects.

\section{Results}

A disappointing number of subjects failed to complete the study protocol. However, the resulting groups were well matched according to age, height and weight. The group performing GPT was smaller in number and included the only two women who completed the study.

\section{Pulmonary function tests}

Initial measurements showed a similar degree of airflow obstruction for the two groups (Table 2). The group having IMT showed an increase in $\mathrm{FEV}_{1}$ from $0.83(0.10) \mathrm{L}$ to $0.94(0.13) \mathrm{L}$ after eight weeks $(\mathrm{P}<0.05)$. A similar but statistically insignificant trend was noted in the GMT group. Resting arterial carbon dioxide tension $\left(\mathrm{PaCO}_{2}\right)$ fell from $4.9(0.3) \mathrm{kPa}$ to $4.2(0.2) \mathrm{kPa}$ after eight weeks of GPT $(\mathrm{P}<0.05)$. Other respiratory function variables including lung volumes, gas transfer and $\mathrm{PaO}_{2}$ did not change in either group. Carboxyhemoglobin levels were similar and increased in both groups at the end of the training period $(\mathrm{P}<0.05)$.

\section{Six-minute walking tests}

Both GPT and IMT led to an increase in $\mathrm{WD}_{6}$ (Table 3 and Figure 2). With GPT the mean increase was $32 \mathrm{~m}(6.9 \%$, $\mathrm{P}<0.05)$, whereas with IMT the mean increase was $23 \mathrm{~m}$ $(4.8 \%, \mathrm{P}<0.05)$. The observed differences did not achieve statistical significance until eight weeks of training. Unpaired t-tests showed no differences between the GPT and IMT groups.

\section{Incremental exercise tests}

After eight weeks of training, we were unable to detect significant changes in $\dot{W} \max \dot{V} O_{2} \max , \dot{V}_{E} \max$, the slope of the relationship between expired ventilation and oxygen uptake $\left(\dot{\mathbf{V}}_{\mathbf{E}} / \dot{\mathbf{V}}_{2}\right), f_{c}$ max and the slope of the relationship between heart rate and oxygen uptake $\left(f_{c} / \dot{V} O_{2}\right)$ in either the GPT or IMT groups (Table 4). Also, there were no changes in arterial oxygen saturation at the end of exercise, in either group.

\section{Maximal respiratory pressures}

Changes in maximum static mouth pressures were not detected in either group after eight weeks of training. In the group undergoing GPT, $P_{E}$ max was 89 (14) $\mathrm{cm} \mathrm{H}_{2} \mathrm{O}$ before training and $84(14) \mathrm{cm} \mathrm{H}_{2} \mathrm{O}$ after training; whereas $P_{I}$ max was $52(6) \mathrm{cm} \mathrm{H}_{2} \mathrm{O}$ before training and $64(11) \mathrm{cm} \mathrm{H}_{2} \mathrm{O}$ after training. In the group undergoing IMT, $P_{E}$ max was 94 (11) $\mathrm{cm} \mathrm{H}_{2} \mathrm{O}$ before training and $104(15) \mathrm{cm} \mathrm{H}_{2} \mathrm{O}$ after training; whereas $P_{I} \max$ was $60(7) \mathrm{cm} \mathrm{H}_{2} \mathrm{O}$ before training and $65(8) \mathrm{cm} \mathrm{H}_{2} \mathrm{O}$ after training.

\section{Symptom assessments}

There was a significant reduction in breathlessness, assessed by VAS immediately following 6-minute walking tests in the group undergoing GPT (P $<0.05$, Table 3 and Figure 3$)$. For most subjects, the change was accompanied by a reduction in perceived exertion on the Borg scale, although the mean difference for the group did not achieve statistical significance ( $\mathrm{P}=0.075$, Table 3 ). By contrast, changes in breathlessness and perceived exertion were not detected at the end of the incremental exercise tests on the cycle ergometer for the 
Table 2 Pulmonary function in the two groups

\begin{tabular}{|c|c|c|c|c|c|c|}
\hline & \multicolumn{3}{|c|}{ General physical training } & \multicolumn{3}{|c|}{ Inspiratory muscle training } \\
\hline & Baseline & 4 weeks & 8 weeks & Baseline & 4 weeks & 8 weeks \\
\hline $\mathrm{FEV}_{1}(\mathrm{~L})$ & $0.84(0.13)$ & $0.89(0.16)$ & $0.89(0.16)$ & $0.83(0.10)$ & $0.87(0.11)$ & $0.94(0.13)^{*}$ \\
\hline FVC (L) & $2.06(0.20)$ & $2.04(0.15)$ & $2.08(0.20)$ & $2.18(0.18)$ & $2.25(0.3 \mathrm{I})$ & $2.47(0.28)$ \\
\hline TLC (L) & $6.8(0.5)$ & - & $5.9(0.6)$ & $7.9(0.4)$ & - & $8.1(0.4)$ \\
\hline FRC (L) & $5.3(0.5)$ & - & $4.7(0.4)$ & $6.6(0.4)$ & - & $6.2(0.6)$ \\
\hline $\mathrm{RV}(\mathrm{L})$ & $4.7(0.5)$ & - & $4.4(0.6)$ & $5.5(0.4)$ & - & $5.5(0.5)$ \\
\hline 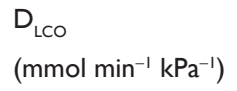 & $3.76(0.65)$ & - & $3.47(0.5 \mathrm{I})$ & $3.6 I(0.55)$ & - & $3.72(0.69)$ \\
\hline $\mathrm{PaO}_{2}(\mathrm{kPa})$ & II.I (0.8) & - & I I.2(0.8) & $10.4(0.8)$ & - & II.I (0.5) \\
\hline $\mathrm{PaCO}_{2}(\mathrm{kPa})$ & $4.9(0.3)$ & - & $4.2(0.2)^{*}$ & $4.4(0.5)$ & - & $4.1(0.2)$ \\
\hline $\mathrm{HbCO}(\%)$ & $2.2(0.3)$ & - & $3.7(0.5)^{*}$ & $1.9(0.5)$ & - & $3.0(0.6)^{*}$ \\
\hline
\end{tabular}

Notes: Values are means (SEM). Statistical significance: *P $<0.05$ compared with baseline.

Abbreviations: $\mathrm{D}_{\mathrm{LCO}}$, carbon monoxide diffusing capacity; $\mathrm{FEV}$, forced expiratory volume in one second; $\mathrm{FVC}$, forced vital capacity; $\mathrm{FRC}$, functional residual capacity; $\mathrm{HbCO}$, carbon dioxide in hemoglobin; $\mathrm{PaCO}_{2}$, arterial carbon dioxide tension; $\mathrm{PaO}_{2}$, arterial oxygen tension; $\mathrm{RV}$, residual volume; TLC, total lung capacity.

group having GPT. After eight weeks of IMT, there were no detectable changes in breathlessness or perceived exertion for either form of exercise assessment.

\section{Discussion}

The selection of subjects for this study was intended to identify those whose exercise capacity was limited directly or indirectly as a result of respiratory impairment and those in whom the degree of airflow obstruction was unlikely to vary with medication, exercise activity, time of day or climatic factors. An improvement in exercise capacity in these subjects could be then more confidently attributed to the effects of the training program.

The 6-minute walking tests revealed two important changes in exercise performance; firstly in the distance walked and secondly in the sensation of dyspnea which accompanied these tests. Both GPT and IMT resulted in increases in 6-minute walking distance. Although statistically significant, the magnitude of these improvements is small (32 $\mathrm{m}$ or $6.9 \%$ for GPT and $23 \mathrm{~m}$ or $4.8 \%$ for IMT). The 6-minute walk is generally believed to be representative of activities of daily living such as shopping, however, the level of exertion associated with this type of test is also considered to be maximal. ${ }^{25}$ Even modest increases in walking distance could therefore translate into clinically meaningful improvements in quality of life, especially if accompanied by improvement in symptoms. There is a recognized learning effect when COPD subjects perform repetitive walking tests. ${ }^{15}$ In accordance with recent recommendation, our subjects performed at least two practice walks to overcome this effect. ${ }^{25}$ Therefore, we can conclude that the subsequent changes in distance walked resulted from the training programs. Improved walking distance after structured walking training is consistent with a true physiological training effect, ie, postponing lactic acidosis and thereby perhaps reducing ventilatory requirement for a given level of exercise. ${ }^{26}$ Since the IMT group did not have any structured physical exercise, the improved walking performance in this group presumably occurred due to different mechanisms such as improved ventilatory capacity or improved ventilatory muscle efficiency. ${ }^{27}$ We can only surmise these effects because we did not attempt to measure blood lactate levels during exercise or assess respiratory muscle endurance or efficiency by direct measurement. Interestingly, Mador and colleagues ${ }^{28}$ did show improved

Table 3 Distance walked (WD $)$, rating of perceived exertion (RPE) and breathlessness scores for the six-minute walking tests

\begin{tabular}{|c|c|c|c|c|c|c|}
\hline & \multicolumn{3}{|c|}{ General physical training } & \multicolumn{3}{|c|}{ Inspiratory muscle training } \\
\hline & Baseline & 4 weeks & 8 weeks & Baseline & 4 weeks & 8 weeks \\
\hline$W D_{6}(m)$ & $462(22)$ & $479(27)$ & $494(33)^{*}$ & 477 (29) & $480(3 I)$ & $500(28)^{*}$ \\
\hline RPE (6-20) & I3.4 (I.I) & II.6 (0.4) & $11.0(0.6)$ & I $4.0(I . I)$ & $13.3(0.9)$ & I $3.8(0.7)$ \\
\hline Dyspnea $(1-100)$ & $53(8)$ & $33(6)^{*}$ & $32(5)^{*}$ & $65(9)$ & $56(9)$ & $61(6)$ \\
\hline
\end{tabular}

Notes: Values are means (SEM). Statistical significance: *P $<0.05$ compared with baseline. 


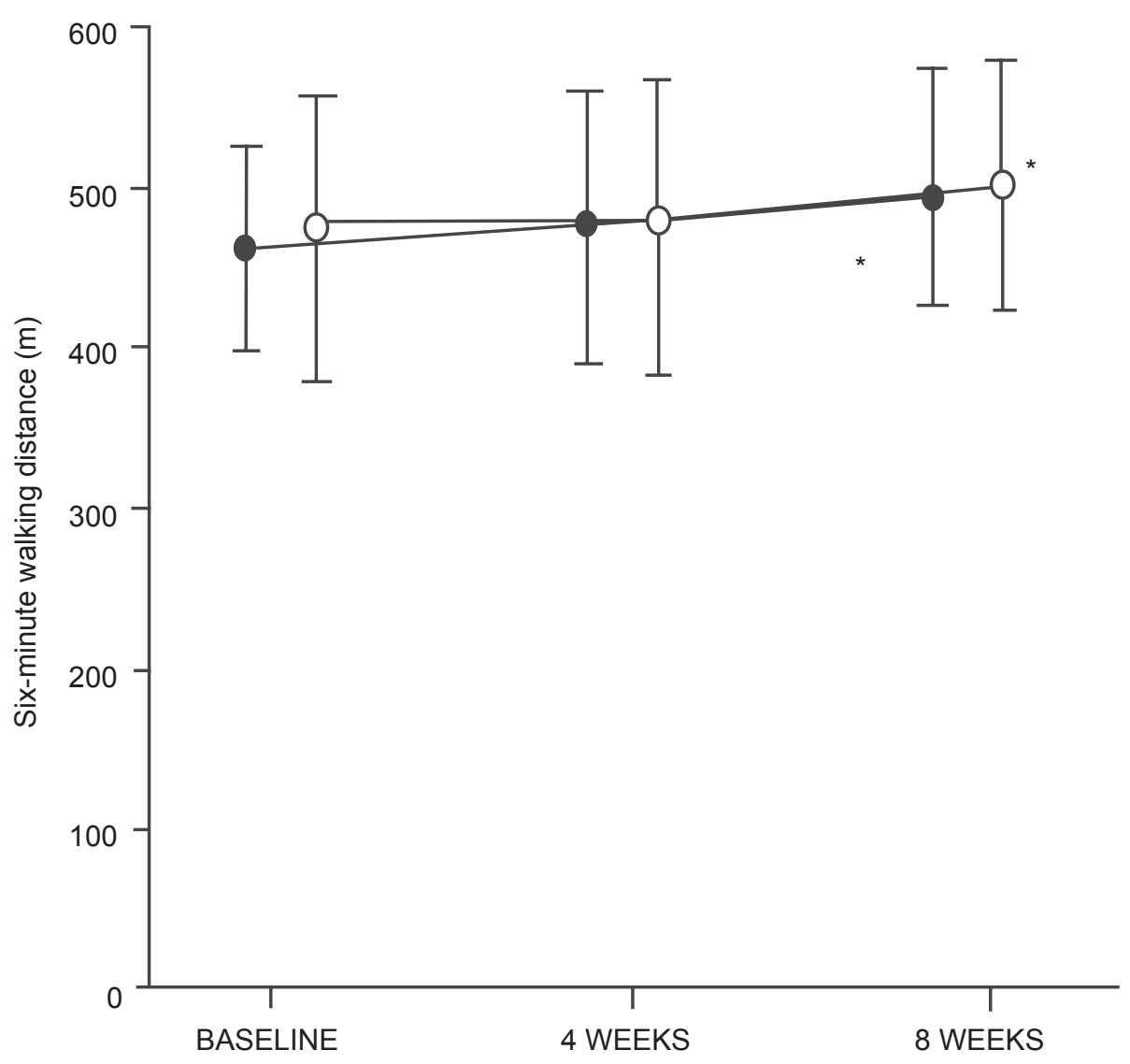

Figure 2 Six-minute walking distances before, during and after eight weeks of general physical training $(\lambda-\lambda, n=7)$ or respiratory muscle training $(\mu-\mu, n=9)$. Mean values are shown with standard error bars. An increase of $6.9 \%$ occurred after general physical training $(n=7)$ and an increase of $4.8 \%$ followed respiratory muscle training ( $n=9$ ).

Note: Statistical significance: $* \mathrm{P}<0.05$.

ventilatory muscle endurance and strength after training by voluntary hyperventilation, however, these adaptations were not accompanied by significant improvements in exercise performance or quality of life. A recent meta-analysis ${ }^{29}$ of inspiratory muscle training using resistive and threshold loading suggested that training was more effective in those with documented inspiratory muscle weakness $\left(P_{I} \max <60 \mathrm{~cm}\right.$ $\left.\mathrm{H}_{2} \mathrm{O}\right)$ and also in those who exhibited ventilatory limitation during exercise, however in these subgroups in our study we found no evidence in support of these suggestions.

The group having GPT were less dyspneic during walking tests after training although a significant change in perceived

Table 4 Selected data from incremental exercise tests

\begin{tabular}{|c|c|c|c|c|c|c|}
\hline & \multicolumn{3}{|c|}{ General physical training } & \multicolumn{3}{|c|}{ Inspiratory muscle training } \\
\hline & Baseline & 4 weeks & 8 weeks & Baseline & 4 weeks & 8 weeks \\
\hline Wmax (watt) & $49(9)$ & $51(10)$ & $58(10)$ & $52(9)$ & $57(8)$ & $57(8)$ \\
\hline $\mathrm{VO}_{2 \max }\left(1 \mathrm{~min}^{-1}\right)$ & $0.91(0.09)$ & $0.87(0.14)$ & $0.99(0.16)$ & $0.84(0.09)$ & $0.86(0.09)$ & $0.98(0.19)$ \\
\hline $\mathrm{V}_{\mathrm{Emax}}\left(I \min ^{-1}\right)$ & $29.4(3.5)$ & $28.2(4.5)$ & $32.8(5.5)$ & $28.6(3.2)$ & $29.7(3.2)$ & $30.0(3.2)$ \\
\hline $\mathrm{V}_{\mathrm{E}} / \mathrm{VO}_{2}$ & $24.8(1.8)$ & $21.3(3.3)$ & $26.7(2.2)$ & $27.6(3.8)$ & $29.1(3.9)$ & $27.3(3.6)$ \\
\hline$f_{C_{\max }}\left(\min ^{-1}\right)$ & $115(8)$ & $116(8)$ & $120(0.9)$ & $125(5)$ & $125(6)$ & $127(5)$ \\
\hline $\mathrm{f}_{\mathrm{C}} / \mathrm{VO}_{2}\left(\mathrm{I}^{-1}\right)$ & $35.1(6.5)$ & $52.8(11.9)$ & $40.6(8.9)$ & $45.0(4.5)$ & $37.9(2.3)$ & $40.2(5.4)$ \\
\hline $\mathrm{SaO}_{\text {2end }}(\%)$ & $91.3(0.8)$ & $91.1(1.0)$ & $91.2(0.9)$ & $90.1(1.5)$ & $91.3(1.4)$ & $91.7(1.9)$ \\
\hline RPE (6-20) & $13.0(1.1)$ & I3.3 (0.9) & I $2.7(1.2)$ & $15.2(0.7)$ & $14.9(0.8)$ & $14.6(0.8)$ \\
\hline Dyspnea $(I-100)$ & $53(7)$ & $50(10)$ & $52(10)$ & $75(5)$ & $67(7)$ & $67(7)$ \\
\hline
\end{tabular}

Note: Values are means (SEM).

Abbreviations: $\mathrm{f}_{\mathrm{Cmax}}$, maximum heart rate; $\mathrm{f}_{\mathrm{C}} / \mathrm{VO}_{2}$, chronotropic index; $\mathrm{RPE}$, rating of perceived exertion; $\mathrm{SaO}_{2 \text { end }}$, oxyhemoglobin saturation at end-exercise; $\mathrm{VO}_{2 \text { max }}$, maximum oxygen uptake; $\mathrm{V}_{\mathrm{Emax}}$ maximum ventilation; $\mathrm{V}_{\mathrm{E}} \mathrm{NO}_{2}$,"slope" of minute ventilation related to oxygen uptake. 


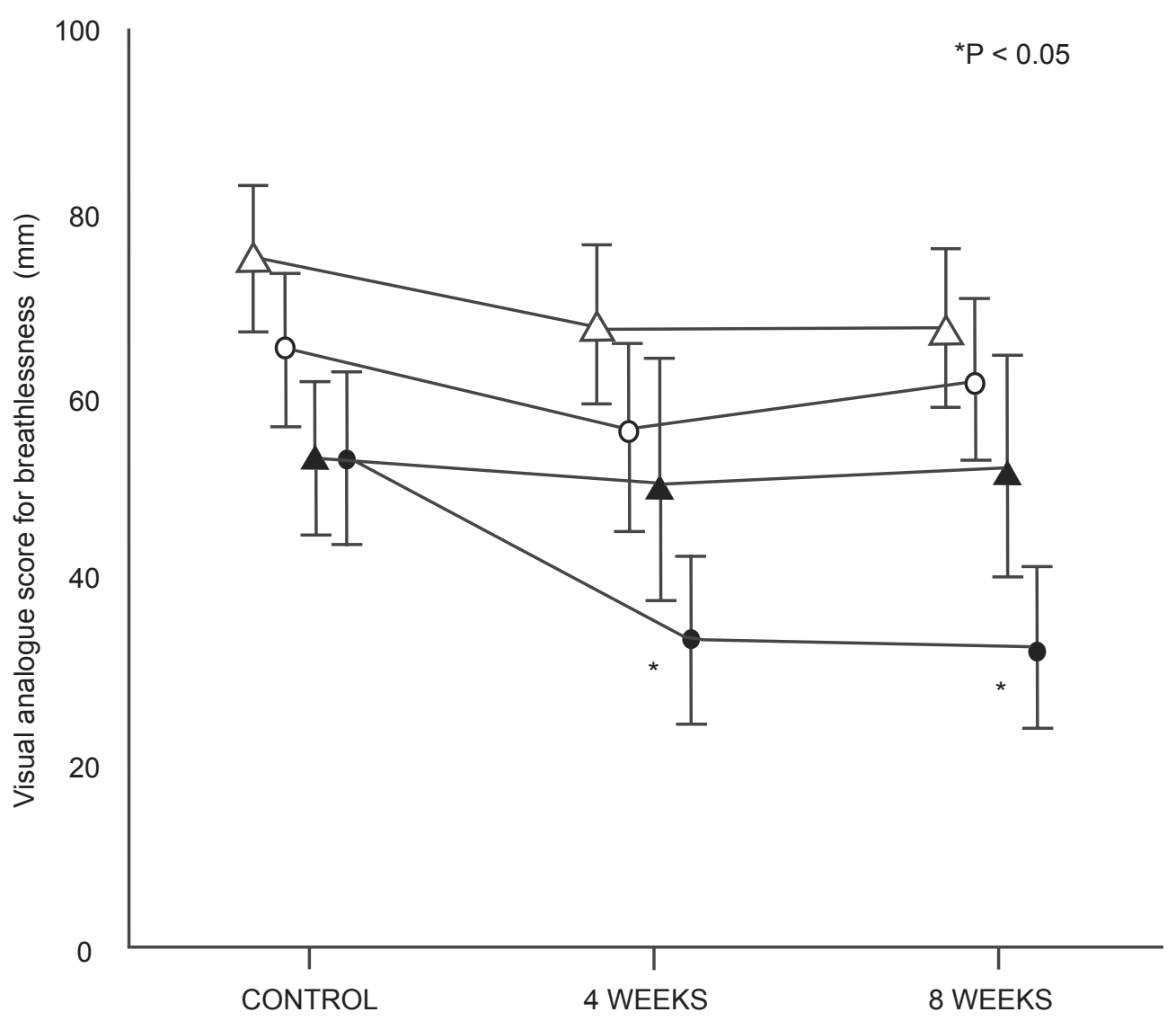

Figure 3 Visual analogue scores for breathlessness on exercise before, during and after eight weeks of general physical training $(\lambda-\lambda$ walking, $\mu-\mu$ cycling, $n=7)$ or respiratory muscle training ( $\boldsymbol{\Delta}-\mathbf{\Delta}$ walking, $\Delta--\Delta$ cycling, $\mathrm{n}=9$ ).

Note: Statistical significance: $* \mathrm{P}<0.05$ compared with baseline.

exertion was not detected. By contrast the group having IMT were equally dyspneic during walking tests before and after training. We feel that this observation is unlikely to have arisen by chance because the effect was seen after both four and eight weeks of training in the same group. It is interesting that both groups of subjects when asked to perform incremental cycle exercise tests to maximum capacity, achieved the same levels of dyspnea at equivalent work rates and levels of ventilation, regardless of training. One explanation for our findings is that COPD patients, encouraged to perform a particular mode of exercise, repetitively, under supervision, become desensitized to dyspnea for that mode of exercise. ${ }^{9}$ Our observation suggests that desensitization to dyspnea occurred specifically during the mode of exercise which was used during training. Stribos and colleagues ${ }^{30}$ found lower dyspnea ratings on a Borg scale at the same work rate during incremental cycle exercise in 15 COPD patients who underwent 12 weeks of home-based rehabilitation. However, in this study the mode of rehabilitative exercise training was not specified. If cycle training was included, then their results would also be consistent with exercise mode-specific desensitization to dyspnea. Other investigators have presented evidence of desensitization to dyspnea as a mechanism of pulmonary rehabilitation. ${ }^{1,9,10,31,32}$ Reardon and colleagues, ${ }^{31}$ using treadmill exercise in COPD patients, reported lower dyspnea indices at the same $\dot{V}_{E} \max$ and $\dot{V} O_{2}$ max . Their subjects participated in a six-week program of outpatient pulmonary rehabilitation including treadmill and cycle endurance training. O'Donnell and colleagues ${ }^{32}$ reported lower dyspnea indices in relation to $\dot{V}_{E}$ and $\dot{\mathbf{v O}}_{2}$ for incremental cycle exercise in COPD subjects who had multimodality endurance exercise training which included cycle ergometry. These studies clearly demonstrate modifications of the relationship between dyspnea and $\dot{V}_{E}$ or $\dot{\mathbf{V O}}_{2}$ which could be called desensitization with respect to those particular stimuli. Desensitization implies a reduction in perception for a given stimulus and therefore requires knowledge or quantification of the stimulus. ${ }^{33}$ The conclusions we can draw from our study are limited by the fact that we did not directly measure $\dot{V}_{E}$ and $\dot{\mathbf{v O}}_{2}$ during the walking tests. In our subjects, dyspnea could have been reduced because $\dot{V}_{E}$ and $\dot{V} O_{2}$ were lower during the walking tests without 
implying any alteration in the relationship between dyspnea and these variables. However, the stimulus to dyspnea is complex and multi-factorial including musculoskeletal and chemoreceptive components as well as ventilation. If we compare breathlessness scores in relation to the external work performed, we see that dyspnea was less for an increased walking distance. In this context, it is justifiable to conclude that desensitization occurred.

Alteration in the perception of dyspnea could be an effect which is independent of structured physical training. Belman and colleagues ${ }^{34}$ demonstrated progressive reductions in dyspnea rating, using a Borg scale, in COPD patients performing four submaximal treadmill exercise tests over 10 days. Their subjects had no form of exercise reconditioning and physiological indices such as $\dot{V}_{E}$ and $\dot{\mathbf{V}}_{2}$ were unchanged for equivalent work rates. Knox and colleagues, ${ }^{15}$ in a study of reproducibility of walking tests also reported improvements in visual analog scores for dyspnea over three days of repeated testing but not over four weeks. These investigators suggested that the reduced dyspnea was part of a learning effect or related to changing attitudes and beliefs which are known to be strong predictors of exercise performance. ${ }^{35}$ Our subjects performed two practice walking tests and familiarized themselves with the psychometric scales to overcome any such learning effects. Furthermore, we did not observe reductions in dyspnea for subjects tested by cycle ergometry. We believe, therefore, that the reductions in dyspnea during walking tests after walking training truly represent a exercise mode-specific desensitization.

In normal subjects, regular physical training results in a physiological training response characterized by increased work capacity ( $\dot{\mathbf{W}} \max$ ), increased $\dot{\mathbf{v O}}_{2} \max$, delayed accumulation of lactic acid and reduced levels of ventilation and heart rate in relation to work intensity $\left(\dot{\mathbf{V}}_{\mathbf{E}} / \dot{\mathbf{V O}}_{2}\right.$ and $\left.\mathbf{f}_{\mathbf{c}} / \dot{\mathbf{V O}}_{2}\right) \cdot{ }^{36,37}$ In our study, neither GPT nor IMT provided evidence of a physiological training effect by any of the above criteria. Increases in $\dot{\mathbf{V O}}_{2}$ max of $8.9 \%$ after GPT and $16.7 \%$ after IMT were observed but these failed to achieve statistical significance. This could represent a type-2 statistical error and with larger numbers of subjects a significant difference might have been detected. Our results do concur with those of Pardy and colleagues, ${ }^{38}$ who found increases in $\dot{\mathbf{V O}}_{2}$ max of similar magnitude in nine COPD patients after physical training and also with Sonne and Davis $^{39}$ who showed a $17 \%$ increase in $\dot{V}_{E}$ max and a $15 \%$ increase in $\mathbf{V O}_{2} \max$ in six patients completing a physical training program. Our inability to detect a physiological training effect could indicate that the training intensity was insufficient. When training intensities are higher (eg $>80 \%$ of $\dot{\mathbf{V}} \mathbf{O}_{2} \max$ ) and closely monitored, increases in $\dot{W} \max$ and $\dot{\mathbf{v}} \mathbf{O}_{2}$ max have been observed in similar patients. ${ }^{40}$

We were unable to demonstrate any improvement in respiratory muscle strength as a result of IMT. However, inspiratory resistive loading is a form of respiratory muscle endurance training and might not, according to known specificity of training effects, lead to demonstrable changes in maximal respiratory pressures. Several studies have reported improvements in respiratory muscle endurance with resistive breathing or voluntary hyperpnea. ${ }^{8,10,41}$ However, a meta-analysis of 17 studies failed to reveal improvements in exercise performance. ${ }^{7}$ It seems that exercise training per se is more likely to improve respiratory muscle performance than laboratory methods for IMT. Two particular studies failed to show a change in $\dot{V} O_{2}$ max after inspiratory resistance training. ${ }^{41,42}$ Another study, in which patients were assessed by stair-climbing, showed no advantage of inspiratory resistance training over sham resistances. ${ }^{43}$

A major disadvantage of this study was the small numbers of subjects completing the experimental protocol. Due to the high rate of attrition we did not have enough subjects to meet the target set by our a priori power analysis. Consequently, our results are susceptible to a type- 2 statistical error and the negative findings must be judged accordingly. Nevertheless, the positive findings from this study, ie the increased walking distance and reduction in dyspnea for walking training, are convincing despite the small numbers of subjects. The withdrawal of $48 \%$ of subjects was disappointing but the difficulty in retaining patients in outpatient programs of rehabilitation is well recognized. ${ }^{44}$ Clearly the frequency of exacerbations of COPD, seen in $19 \%$ of these subjects, has a disruptive effect on planned training programs. The voluntary withdrawal of another $19 \%$ reflects the difficulty in sustaining motivation of subjects with disabling COPD.

The results of a comparison between general physical training and respiratory muscle training, each supervised for eight weeks in small groups of COPD subjects, can be summarized as follows: Both groups increased exercise capacity for a standardized walking test. The average improvement was less than $10 \%$ but nevertheless statistically significant and likely to be clinically meaningful in COPD patients. Walking exercise after GPT was associated with less dyspnea but this was not the case after IMT. By contrast, dyspnea at the end of symptom-limted, incremental exercise tests on a cycle ergometer was unchamged despite GPT or IMT. These findings are consistent with desensitization to dyspnea that is specific for the type of exercise training program. 


\section{Acknowledgments}

This work constitutes part of an MD thesis which was supported by a grant from West Lambeth Health Authority Research Committee (Endowments). I wish to thank Cathy Smith from the Department of Physiotherapy at St. Thomas' Hospital, London for her supervision of the subjects undergoing general physical training. I would also like to express appreciation to Professor Ian R Cameron for his guidance in the conduct of this study.

\section{References}

1. Cockcroft AE, Saunders MJ, Berry G. Randomised controlled trial of rehabilitation in chronic respiratory disability. Thorax. 1981;36:200-3.

2. Griffiths TL, Burr ML, Campbell IA, et al. Results at 1 year of outpatient multidisciplinary pulmonary rehabilitation: A randomised controlled trial. Lancet. 2000;355:362-8.

3. Casaburi R, Patessio A, Ioli F, et al. Reductions in exercise lactic acidosis and ventilation as a result of exercise training in patients with obstructive lung disease. Am Rev Respir Dis. 1991;143:9-18.

4. Casaburi R, Porszasz J, Burns MR, et al. Physiologic benefits of exercise training in rehabilitation of patients with severe chronic obstructive pulmonary disease. Am J Respir Crit Care Med. 1997;155:1541-51.

5. Pitta F, Troosters T, Spruit MA, et al. Characteristics of physical activities in daily life in chronic obstructive pulmonary disease. Am J Respir Crit Care Med. 2005;171:972-7.

6. Cooper CB. Determining the role of exercise in patients with chronic pulmonary disease. Med Sci Sports Exerc. 1995;27:147-57.

7. Smith K, Cook D, Guyatt GH, et al. Respiratory muscle training in chronic airflow limitation: A meta-analysis. Am Rev Respir Dis. 1992;145:533-9.

8. Dekhuijzen PN, Folgering HT, van Herwaarden CL. Target-flow inspiratory muscle training during pulmonary rehabilitation in patients with copd. Chest. 1991;99:128-33.

9. Agle DP, Baum GL, Chester EH, et al. Multidiscipline treatment of chronic pulmonary insufficiency. 1. Psychologic aspects of rehabilitation. Psychosom Med. 1973;35:41-9.

10. Levine S, Weiser P, Gillen J. Evaluation of a ventilatory muscle endurance training program in the rehabilitation of patients with chronic obstructive pulmonary disease. Am Rev Respir Dis. 1986;133:400-6.

11. Definition and classification of chronic bronchitis for clinical and epidemiological purposes. A report to the Medical Research Council by their Committee on the Aetiology of Chronic Bronchitis. Lancet. 1965;1:775-9.

12. Standardized lung function testing. Report working party. Bull Eur Physiopathol Respir. 1983;19 Suppl 5:1-95.

13. ATS Committee on Proficiency Standards for Clinical Pulmonary Function Laboratories. ATS statement: Guidelines for the six-minute walk test. Am J Respir Crit Care Med. 2002;166:111-7.

14. Guyatt GH, Pugsley SO, Sullivan MJ, et al. Effect of encouragement on walking test performance. Thorax. 1984;39:818-22.

15. Knox AJ, Morrison JF, Muers MF. Reproducibility of walking test results in chronic obstructive airways disease. Thorax. 1988;43:388-92.

16. Buchfuhrer MJ, Hansen JE, Robinson TE, et al. Optimizing the exercise protocol for cardiopulmonary assessment. J Appl Physiol. 1983;55:1558-64

17. Cooper CB, Storer TW. Exercise testing and interpretation: A practical approach. New York: Cambridge University Press; 2001.

18. Shephard RJ, Allen C, Benade AJ, et al. The maximum oxygen intake. An international reference standard of cardiorespiratory fitness. Bull World Health Organ. 1968;38:757-64.
19. Wilson SH, Cooke NT, Edwards RH, et al. Predicted normal values for maximal respiratory pressures in caucasian adults and children. Thorax. 1984;39:535-8.

20. Borg G. Perceived exertion as an indicator of somatic stress. Scand J Rehabil Med. 1970;2:92-8.

21. Silverman M, Barry J, Hellerstein H, et al. Variability of the perceived sense of effort in breathing during exercise in patients with chronic obstructive pulmonary disease. Am Rev Respir Dis. 1988;137:206-9.

22. Mador MJ, Kufel TJ. Reproducibility of visual analog scale measurements of dyspnea in patients with chronic obstructive pulmonary disease. Am Rev Respir Dis. 1992;146:82-7.

23. Maxwell C. Sensitivity and accuracy of the visual analogue scale: A psycho-physical classroom experiment. Br J Clin Pharmacol. 1978;6:15-24.

24. Altman DG, Gore SM, Gardner MJ, et al. Statistical guidelines for contributors to medical journals. $\mathrm{Br}$ Med J (Clin Res $\mathrm{Ed}$ ). 1983;286:1489-93.

25. Kervio G, Carre F, Ville NS. Reliability and intensity of the sixminute walk test in healthy elderly subjects. Med Sci Sports Exerc. 2003;35:169-74.

26. Casaburi R, Storer TW, Wasserman K. Mediation of reduced ventilatory response to exercise after endurance training. J Appl Physiol. 1987;63:1533-8.

27. Belman MJ, Shadmehr R. Targeted resistive ventilatory muscle training in chronic obstructive pulmonary disease. J Appl Physiol. 1988;65:2726-35.

28. Mador MJ, Deniz O, Aggarwal A, et al. Effect of respiratory muscle endurance training in patients with copd undergoing pulmonary rehabilitation. Chest. 2005;128:1216-24.

29. Lotters F, van Tol B, Kwakkel G, et al. Effects of controlled inspiratory muscle training in patients with copd: A meta-analysis. Eur Respir J. 2002;20:570-6.

30. Strijbos JH, Postma DS, van Altena R, et al. Feasibility and effects of a home-care rehabilitation program in patients with chronic obstructive pulmonary disease. J Cardiopulm Rehabil. 1996;16:386-93.

31. Reardon J, Awad E, Normandin E, et al. The effect of comprehensive outpatient pulmonary rehabilitation on dyspnea. Chest. 1994;105:1046-52.

32. O'Donnell DE, McGuire M, Samis L, et al. The impact of exercise reconditioning on breathlessness in severe chronic airflow limitation. Am J Respir Crit Care Med. 1995;152:2005-13.

33. Killian KJ. The objective measurement of breathlessness. Chest. 1985;88:84S-90S.

34. Belman MJ, Brooks LR, Ross DJ, et al. Variability of breathlessness measurement in patients with chronic obstructive pulmonary disease. Chest. 1991;99:566-71.

35. Morgan AD, Peck DF, Buchanan DR, et al. Effect of attitudes and beliefs on exercise tolerance in chronic bronchitis. $\mathrm{Br}$ Med J (Clin Res Ed). 1983;286:171-3.

36. Casaburi R, Storer TW, Ben-Dov I, et al. 1987. Effect of endurance training on possible determinants of vo2 during heavy exercise. $\mathrm{J} \mathrm{Appl}$ Physiol. 1987;62:199-207.

37. Edwards RH, Jones NL, Oppenheimer EA, et al. Interrelation of responses during progressive exercise in trained and untrained subjects. Q J Exp Physiol Cogn Med Sci. 1969;54:394-403.

38. Pardy RL, Rivington RN, Despas PJ, et al. Inspiratory muscle training compared with physiotherapy in patients with chronic airflow limitation. Am Rev Respir Dis. 1981;123:421-5.

39. Sonne LJ, Davis JA. Increased exercise performance in patients with severe copd following inspiratory resistive training. Chest. 1982;81:436-9.

40. Punzal PA, Ries AL, Kaplan RM, et al. Maximum intensity exercise training in patients with chronic obstructive pulmonary disease. Chest. 1991;100:618-23.

41. Chen H, Dukes R, Martin BJ. Inspiratory muscle training in patients with chronic obstructive pulmonary disease. Am Rev Respir Dis. 1985;131:251-5. 
42. Jones DT, Thomson RJ, Sears MR. Physical exercise and resistive breathing training in severe chronic airways obstruction - are they effective? Eur J Respir Dis. 1985;67:159-66.

43. Bjerre-Jepsen K, Secher NH, Kok-Jensen A. Inspiratory resistance training in severe chronic obstructive pulmonary disease. Eur J Respir Dis. 1981;62:405-11.
44. Jederlinic P, Muspratt JA, Miller MJ. Inspiratory muscle training in clinical practice. Physiologic conditioning or habituation to suffocation? Chest. 1984;86:870-3. 
